\title{
Cognitive masking in rapid sequential processing: The effect of an emotional picture on preceding and succeeding pictures*
}

\author{
MATTHEW HUGH ERDELYI and DONNA G. BLUMENTHAL \\ Douglass College, Rutgers University, New Brunswick, New Jersey 08903
}

\begin{abstract}
This experiment assessed the effect of an emotional pictorial input on the processing of preceding and succeeding pictorial inputs. Thirty-nine individual pictures were successively presented for $165 \mathrm{msec}$, with a $500-\mathrm{msec}$ dark interval between each item. The 20th input item was either an emotional or a neutral picture; the remaining 38 items were neutral sketches. Postinput recognition data indicated a processing disruption of several pictorial items following the emotional as compared to the neutral input and a possible processing disruption of the item immediately preceding the emotional stimulus. The outcome suggests nonindependence in the processing of separate picture inputs in rapid sequential presentations.
\end{abstract}

In a recent study, Erdelyi and Appelbaum (1973) demonstrated that, in a single-fixation exposure of an array of pictures, an emotional picture disrupts the processing of neighboring neutral pictures. The effect was labeled "cognitive masking," inasmuch as a cognitive-symbolic aspect of a stimulus element, not just a physical aspect, was shown capable of affecting the processing of topographically contiguous items. The present study was undertaken to determine whether this cognitive masking effect would generalize from topographic to temporal contiguity.

There is already some evidence that sequentially inputted verbal materials can interact inhibitively. Recent reports by Saufley and Winograd (1970), Schultz (1971), and Tulving (1969) demonstrate that a "high-priority event" (e.g., the name of a famous person, such as Aristotle, Freud, etc.) can inhibit the processing of preceding or succeeding items. While interesting, the outcomes are not surprising, given the ubiquitous emphasis in the modern literature of the role of rehearsal in the consolidation of labile memory traces into more permanent memory structures. The high-priority items are presumably accorded extra rehearsal time at the expense of neighboring items, which lead to the expectation of better retention of the high-priority items relative to the low-priority items.

Surprisingly, two recent studies on very rapid sequential processing of pictures raise doubts as to whether pictorial inputs can produce the effects hitherto demonstrated with verbal materials. Specifically, Potter and Levy (1969) and Shaffer and Shiffrin (1971) have shown that recognition memory for rapidly sequenced pictures varies with the items' exposure duration but not with the duration of interitem intervals (potential rehearsal periods) or with success or failure in processing

\footnotetext{
*Support of this research by Grant 07-2120 from the Rutgers University Research Council is gratefully acknowledged.
}

immediately preceding or following items. These data therefore imply an independence between the processing of a given pictorial item and what occurs before or after stimulus exposure, whether this be a blank rehearsal period or the processing of another picture. Such independence would be of particular significance since most everyday visual experiences are pictorial rather than verbal. Given the limitations on the number of items that can be processed per unit time, an independence between the processing of temporally adjacent pictures would in effect imply an inability on the part of the processor to select what elements of the input sequence he will process for storage in long-term memory. Such inability would be a severe blow to the increasingly emergent view of the processor as an active agent, selectively controlling what he processes and stores from the overrich stimulus world (e.g., Lindsay \& Norman, 1972; Moray, 1970; Neisser, 1967).

The present study was undertaken to determine whether a high-priority pictorial item, specifically an emotional item, would have an inhibitory or "amnestic" (Tulving, 1969) effect on the preceding or succeeding items. An inhibitory effect, presumably brought about by a monopolization of processing time (attention) by the critical item, would argue against item independence in sequential picture processing and in favor of item interactions based on stimulus meaning and selective strategies of the processor.

\section{METHOD}

\section{Subjects}

The Ss were 20 male and female undergraduates drawn from a perception laboratory at Douglass College. The Ss were tested in two randomly selected groups of $10 \mathrm{Ss}$ each.

\section{Procedure}

The experimental design involved two groups, both of which 


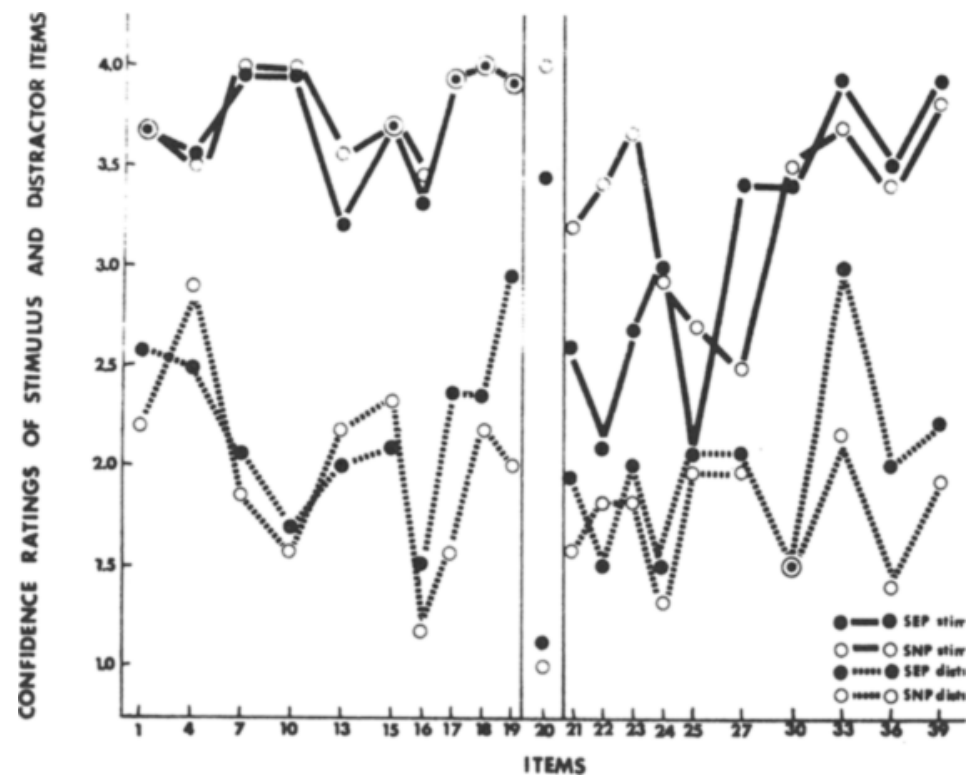

Fig. 1. Average recognition confidence ratings of stimulus items and distractor counterparts for SEP (sketches-emotional picture) and SNP (sketches-neutral picture) Ss.

were exposed to a rapid sequence of 39 individual pictures. Both groups were shown the identical stimuli except for the middle item (the 20th), which for the emotional picture group consisted of a photograph of a horribly deformed child's face, and for the neutral picture group, of a photograph of a normal child's face. Items 1-19 and 21-39, identical for both groups, consisted of simple sketches of easily identifiable objects or animals (e.g., lamp, elephant, tennis racket, key, rat, etc.). The Ss in the two groups, SEP (sketches-emotional picture) and SNP (sketches-neutral picture), were treated identically in all respects except for the critical 20th item.

The rapid sequential presentation of the stimuli was achieved cinematographically. Two black and white super-8 film strips were prepared from slides of the stimuli, containing in sequence the broken chain of 38 sketches (1-19 and 21-39) and either the deformed or the normal face as the 20th item. The order of sketches was randomly generated before the filming of the stimulus sequence and was the same for both film versions. The temporal unit in the film sequence was a movie frame, roughly a 55-msec period. Each item (including the 20th) lasted three frames (approximately $165 \mathrm{msec}$ ). The interitem interval constituted nine dark frames, lasting approximately $500 \mathrm{msec}$. The $1 / 2-\mathrm{sec}$ off interval between items precluded classical backward or forward masking effects arising between items from luminance or contour interactions, since such effects do not operate beyond about $100-\mathrm{msec}$ interstimulus intervals (see, for example, Kahneman, 1968; Raab, 1963; Weisstein \& Haber, 1965). The total presentation time of the 39 items, including the blank periods between them, was about $26 \mathrm{sec}$.

The retention measure following the film was a recognition with ratings procedure. The Ss were shown a series of slides for $10 \mathrm{sec}$ each, half of which were slides of the stimulus items (i.e., items in the film) and half of which were distractor items (slides of comparable pictures not in the film). After viewing each slide, the $\mathrm{Ss}^{\prime}$ task was to write the item's name into a prepared recognition protocol and then rate their recognition confidence on a scale from 1 to 4 . A rating of " 1 " was meant to indicate high confidence that the item was not in the film; a " 4 " rating was meant to indicate an equally high confidence that the item had been in the film; " 3 " and " 4 " ratings were to represent intermediate levels of confidence in either direction.

The order of stimulus items in the test phase was not the same as in the presentation (film) phase. Excepting the critical 20th item, the test order of stimulus items was randomly determined. The 20th input item (the neutral or emotional face) was the penultimate test item, the last test item being a distractor-the emotional face for the SNP group and the neutral face for the SEP group.

Each distractor slide was randomly paired with a stimulus slide. A toss of a coin determined whether each stimulus slide preceded or followed its distractor counterpart. The sequence of stimulus and distractor test items was identical for both the SEP and SNP groups except for the reversal in order of the last two face items in the test list.

So as to reduce fatigue and distractibility, only a subset of the stimulus (film) items was tested for recognition. The items tested, plus their distractor counterparts, were Input Items 1, 4, $7,10,13,15,16,17,18,19,20,21,22,23,24,25,27,30,33$, 36 , and 39 . Since the effect of the emotional stimulus on preceding and subsequent items was the focus of interest, the nearest five preceding and following items from the critical 20th item were included in the test phase.

\section{RESULTS}

Figure 1 presents the average group recognition rating of each tested stimulus item plus the group rating of each distractor counterpart.

Ignoring the distractor ratings for the moment, it will be noted that the two groups' stimulus ratings are exceedingly close up to the critical 20 th item. A very noticeable rating difference between the SEP and SNP groups emerges suddenly following the 20th stimulus item, lasting through the 23rd item. Although a drop in recognition ratings seems to occur for both groups, visual inspection suggests a far more dramatic recognition decrement on Items 21,22 , and 23 following the emotional in comparison to the neutral 20th stimulus input. The two groups' recognition ratings seem to converge beyond the 23 rd input item, with both groups producing what appears to be a bow-shaped serial position effect in the region of Input Orders 24 and 26.

Although the general question posed by this study is relatively straightforward-Does an emotional stimulus inhibit the processing of preceding and subsequent 
Fig. 2. SEP and SNP Ss' average recognition confidence ratings of stimulus items linearly corrected for distractor ratings.

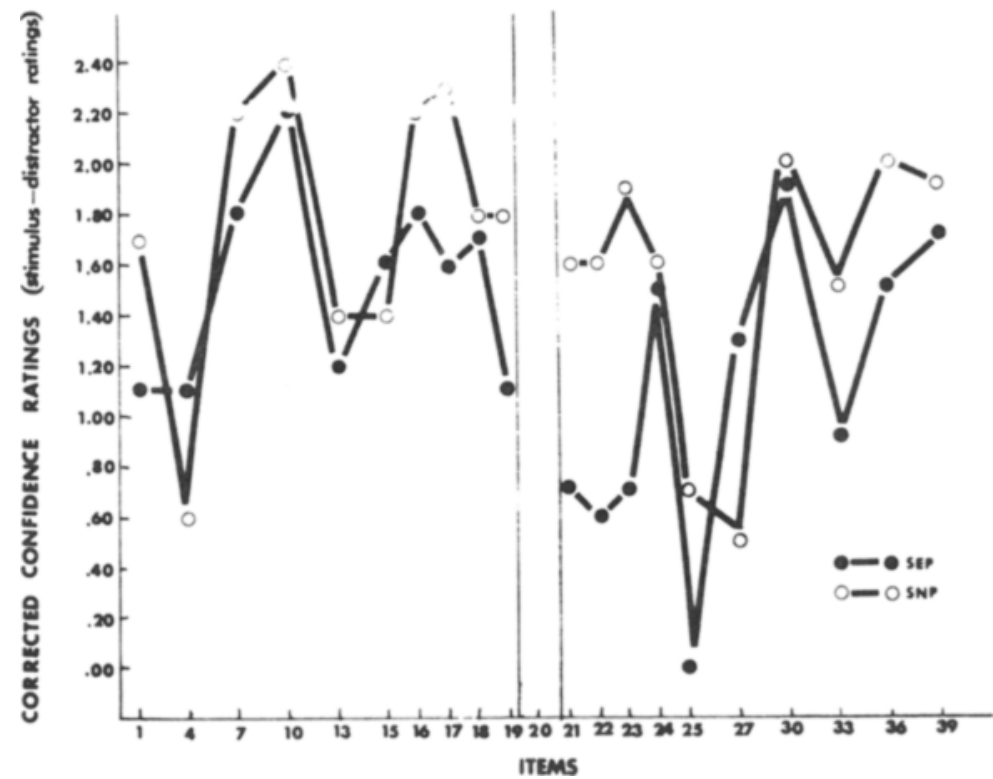

items?-the specific a priori tests to perform on the projected data were by no means obvious to generate. One test which seemed reasonable, particularly since it is the one performed by previous researchers using this type of paradigm, was a comparison of group performance on the items immediately preceding and immediately following the critical event. This is not the only indicated test, however. A temporal lag in even a very powerful effect would not be difficult to imagine, with maximum group divergence occurring not immediately before or after the critical event, but a little time before or a little time later. Clearly, there was no way of anticipating the specific recognition profiles in the region of the critical input, so that some guesswork inevitably entered into the generation of the a priori tests. Two planned comparisons were decided upon. The first was the already mentioned tests of group differences on the items immediately preceding (19th) and immediately following (21st) the critical item (20th). The second a priori test was a more general one which, it was hoped, would be sensitive to more complex overall group differences which the first test might miss: group comparisons of overall performance on the five precritical items (Nos. 15, 16, 17, 18, and 19) and the five postcritical items (Nos. 21, 22, 23, 24, and 25).

Still disregarding the distractor ratings, it is clear from Fig. 1 that no statistical test is required on the precritical items. The two groups' average recognition scores on the immediately preceding item (Item 19) are numerically identical. Overall performance on the five precritical items (Nos. 15, 16, 17, 18, and 19) is virtually identical for both groups. Such exceedingly close recognition performance between the groups on precritical items underscores the sudden divergence observed immediately following the critical item up to and including Item 23 .

A 2 by 5 (Group by Item) analysis of variance on Ss' ratings of Postcritical Items 21, 22, 23, 24, and 25 revealed that the SEP group's overall recognition performance on the five postcritical items was significantly poorer than the SNP group's $[F(1,18)=$ $4.66, \mathrm{p}<.05$ ]. (Item order and the Group by Item interaction were not significant.) A test of the difference of group means on the immediate postcritical item (No. 21) proved marginally significant $[\mathrm{t}(18)=1.34$, $\mathrm{p}<.10]$. Taken together, these data appear to suggest that an emotional pictorial input has a disrupting effect on the processing of subsequent pictorial inputs, but not on the processing of preceding inputs. Inspection of the group profiles in Fig. 1 suggests that the disruption involved the three postcritical inputs (Nos. 21, 22, and 23), with maximum disruption occurring not on the immediately subsequent item (No.21) but on the next two (Items 22 and 23).

Up to this point, the data have been discussed without regard to Ss' performance on the distractor items. The function of the distractor ratings was to check on any major systematic group divergences in rating criteria. While a considerable within-group variability was anticipated on distractor ratings because of differences in confusability with stimulus items, no systematic between-group variability was expected on ratings of the same distractors. Any such major group discrepancies would suggest group differences in rating criteria. Inspection of the distractor data in Fig. 1 reveals the expected within-group variability in distractor ratings. However, with perhaps one significant exception to be discussed further below, the two groups' distractor rating profiles are exceedingly close, suggesting that a correction for possible rating biases should leave the basic result pattern essentially unchanged. Fig. 2 depicts the recognition profiles resulting from a linear correction for bias-where distractor ratings are subtracted from their counterpart stimulus ratings. 
Although more variable, the data pattern of Fig. 2 tends to be consistent with the uncorrected stimulus recognition ratings. Most importantly, corrected recognition performance following the critical event suggests, like the uncorrected data, a drop in the SEP group's recognition of postcritical items relative to the SNP group's [for the five postcritical items, $\mathrm{F}(1,18)=$ $4.14, \mathrm{p} \cong .05$; for the immediate postcritical item, Item $21, t(18)=1.66, p \cong .05]$.

One discrepancy, though it may well be a chance effect, occurs in the region of the precritical items, particularly for the immediate precritical item (No. 19). With stimulus ratings corrected for distractor ratings, what appeared to be identical recognition performance in the uncorrected ratings becomes a possible group difference in recognition of precritical items. The Group by Item analysis of variance on corrected ratings of Precritical Items $15,16,17,18$, and 19 did not reveal a significant group effect $[F(1,18)=2.78]$, but the group comparison on the immediate precritical item did prove significant $[t(18)=2.13, p<.05]$. It is not clear how heavily to weight this discrepant outcome which, unlike the uncorrected data, suggests a backward disruptive effect by the emotional critical item.

Perhaps the best course is to emphasize the overall agreement between the corrected and uncorrected recognition data, particularly on postcritical input performance, and leave open the possibility of a backward cognitive masking effect pending further research.

\section{CONCLUSIONS AND SUMMARY}

The purpose of this study was to determine whether an emotional pictorial input occurring within a sequence of rapid pictorial inputs would disturb the processing of preceding and succeeding pictorial items. Recognition ratings, both corrected as well as uncorrected for criterion effects, indicated that an emotional input depressed retention of several succeeding pictorial inputs. The uncorrected recognition data revealed no backward inhibition effect by the emotional stimulus, but the corrected ratings suggested a possible interference with the pictorial input immediately preceding the emotional stimulus.

Thus, while there is some uncertainty about the emotional input's effect on preceding pictorial items, the data are consistent in suggesting a forward cognitive masking effect by the emotional stimulus. These results, even with the uncertain backward effect discounted, indicate a nonindependence of pictorial items in rapid sequential processing.

Such nonindependence may be understood in the framework of a division of attention paradigm in which a constant and limited attentional capacity is shared between a detection and a central processing task, to the detriment of one or both tasks (Moray, 1970). The emotional input might monopolize not only rehearsal-consolidation processes at the expense of temporally adjacent items, but may well induce high-priority memory consultations concerning the critical input (to recheck, assess, reach decisions about actions to be taken, etc.) which would interfere with the detection and consolidation of temporally neighboring low-priority pictures.

\section{REFERENCES}

Erdelyi, M. H., \& Appelbaum, A. Cognitive masking: The disruptive effect of an emotional stimulus upon the perception of contiguous neutral items. Bulletin of the Psychonomic Society, 1973, 1, 59-61.

Kahneman, D. Method, findings, and theory in studies of visual masking. Psychological Bulletin, 1968, 70, 404-425.

Lindsay, P. H., \& Norman, D. A. Human information processing. New York: Academic Press, 1972.

Moray, N. Attention: Selective processes in vision and hearing. New York: Academic Press, 1970.

Neisser, U. Cognitive psychology. New York: AppletonCentury-Crofts, 1967.

Potter, M. C., \& Levy, E. I. Recognition memory for a rapid sequence of pictures. Journal of Experimental Psychology, $1969,81,10-15$

Raab, D. Backward masking. Psychological Bulletin, 1963, 60, 118-129.

Saufley, W. H., \& Winograd, E. Retrograde amnesia and priority instructions in free recall. Journal of Experimental Psychology, 1970, 85, 150-152.

Schultz, L. S. Effects of high-priority events on recall and recognition of other events. Journal of Verbal Learning \& Verbal Behavior, 1971, 10, 322-330.

Shaffer, W. O., \& Shiffrin, R. M. Rehearsal and storage of visual information. Report No. 71-5, Indiana Mathematical Psychology Program, Indiana University, 1971.

Tulving, E. Retrograde amnesia in free recall. Science, 1969, 164, 88-90.

Weisstein, N., \& Haber, R. N. A U-shaped backward masking function in vision. Psychonomic Science, 1965, 2, 75-76.

(Received for publication October 15, 1972; accepted November 13, 1972.) 